\title{
Rotating black holes in higher order gravity
}

\author{
Christos Charmousis, ${ }^{1}$ Marco Crisostomi, ${ }^{1,2,3}$ Ruth Gregory $\odot{ }^{4,5}$ and Nikolaos Stergioulas ${ }^{6}$ \\ ${ }^{1}$ Laboratoire de Physique Théorique, CNRS, University of Paris-Sud, \\ Université Paris-Saclay, 91405 Orsay, France \\ ${ }^{2}$ Institut de physique théorique, University of Paris Saclay, CEA, CNRS, 91191 Gif-sur-Yvette, France \\ ${ }^{3}$ AIM, CEA, CNRS, University of Paris-Saclay, Univ. Paris Diderot, Sorbonne Paris Cité, \\ F-91191 Gif-sur-Yvette, France \\ ${ }^{4}$ Centre for Particle Theory, Durham University, South Road, Durham, DH1 3LE, United Kingdom \\ ${ }^{5}$ Perimeter Institute, 31 Caroline Street, Waterloo, Ontario N2L 2Y5, Canada \\ ${ }^{6}$ Department of Physics, Aristotle University of Thessaloniki, 54124 Thessaloniki, Greece
}

(Received 3 April 2019; published 11 October 2019)

\begin{abstract}
We develop a new technique for finding black hole solutions in modified gravity that have "stealth" hair, i.e., hair of which the only gravitational effect is to tune the cosmological constant. We consider scalartensor theories in which gravitational waves propagate at the speed of light and show that, subject to a parametric constraint we specify, Einstein metrics can be painted with stealth hair, provided there exists a family of geodesics always normal to spacelike surfaces. We also present a novel scalar-dressed rotating black hole that has finite scalar field at both the black hole and cosmological event horizons.
\end{abstract}

DOI: $10.1103 /$ PhysRevD.100.084020

Black holes are a cornerstone in the study of General Relativity (GR), be it theoretical or practical. From the theoretical perspective, we have several exact solutions corresponding to black holes in GR, with charges such as mass, angular momentum, and even acceleration or Taub Newman-Unti-Tamburino parameters. Of these, the most important is the Kerr solution that describes the rotating black hole; most astrophysical black holes are believed to be rotating, and, indeed, the first detection of gravitational waves was from the merger of two spinning Kerr black holes [1].

Another key objective in gravity is to explain our Universe. One of the main challenges in cosmology is to explain late time acceleration; is it due to dark energy, modified gravity, or a very small cosmological constant $\Lambda$, finely tuned by some as yet undiscovered mechanism? One fruitful means of explaining the small late time acceleration is to modify gravity in such a way as to induce (or tune) a cosmological constant, but if gravity is modified, then it is vital to understand how these modifications affect black holes. If it is not possible to construct astrophysically realistic black hole solutions, then the theory cannot be considered viable.

In GR, the Kerr solution (see Refs. [2,3] for the inclusion of a cosmological constant) describes the most general

Published by the American Physical Society under the terms of the Creative Commons Attribution 4.0 International license. Further distribution of this work must maintain attribution to the author(s) and the published article's title, journal citation, and DOI. Funded by SCOAP. axisymmetric stationary rotating black hole; moreover, it is hoped that the event horizon telescope [4] will soon to be able to directly image the shadow of the black hole at the center of our Galaxy, possibly setting constraints on deviations from Kerr [5]. It is important therefore to investigate whether spinning black hole solutions exist in modified gravity and, if so, whether or not they carry nontrivial extra degrees of freedom.

In this paper, we focus on a particular family of modified scalar-tensor theories of gravity, developing a new technique to find, for the first time, astrophysically realistic rotating black hole solutions with a nontrivial scalar field, or stealth hair. Our method is based on the Hamilton-Jacobi approach to finding geodesics. In brief, we prove that a GR solution can also be a solution to modified gravity if the scalar field is the Hamilton-Jacobi potential for a geodesic congruence in the spacetime in question. This allows for a wide range of physically interesting stealth solutions; in particular, we present examples of rotating black holes with stealth hair.

We focus for definiteness on shift-symmetric scalartensor theories of gravity in the family of degenerate higher order scalar-tensor (DHOST) theories [6-8], although the technique can be generalized to other modifications of gravity. In particular, we will focus on the subset of theories where gravitational waves propagate at the speed of light, $c_{T}=1$, in accord with the recent multimessenger neutron star binary merger observation [9].

The most general shift-symmetric scalar-tensor theory of gravity in which gravitational waves propagate with the speed of light is 


$$
\begin{aligned}
\mathcal{L}= & K(X)+G(X) R+A_{3} \phi^{\mu} \phi_{\mu \nu} \phi^{\nu} \square \phi \\
& +A_{4} \phi^{\mu} \phi_{\mu \rho} \phi^{\rho \nu} \phi_{\nu}+A_{5}\left(\phi^{\mu} \phi_{\mu \nu} \phi^{\nu}\right)^{2}
\end{aligned}
$$

where $K, G$, and $A_{i}$ are all functions of $X=(\partial \phi)^{2}$, and we abbreviate $\partial_{\mu} \phi$ as $\phi_{\mu}$ and $\nabla_{\nu} \nabla_{\mu} \phi$ as $\phi_{\mu \nu}$. In order to propagate a single scalar degree of freedom and avoid Ostrogradski instabilities, $A_{4,5}$ are constrained by

$$
\begin{aligned}
& A_{4}=-A_{3}+\frac{1}{8 G}\left(48 G_{X}^{2}+8 A_{3} G_{X} X-A_{3}^{2} X^{2}\right), \\
& A_{5}=\frac{A_{3}}{2 G}\left(4 G_{X}+A_{3} X\right),
\end{aligned}
$$

where $G_{X}=\partial G / \partial X$. These constraints reduce the number of free functions to 3 ( $K, G$, and $A_{3}$ ), and for simplicity, we do not consider the cubic Horndeski term in our Lagrangian (1), though this can easily be reinstated if required.

A family of exact static spherically symmetric solutions, with the scalar field playing the role of dark energy, was initially found in a class of shift-symmetric Horndeski theories [10]. It has the nice feature of locally describing a Schwarzschild geometry while asymptotically approaching a self-tuned accelerating cosmology. Since these solutions acquire a metric similar to that of GR while having a nontrivial scalar field, they have been widely called stealth solutions. ${ }^{1}$ They can be mapped via disformal transformations to stealth solutions of DHOST theories with unitary speed of gravitational waves [16] and are free of ghost and gradient instabilities [17]. For spherically symmetric stealth solutions in DHOST theories, see Refs. [18-20].

Known stealth solutions are spherically symmetric and all feature the same characteristic: a constant kinetic term for the scalar field that does not deform an underlying Einstein geometry. We suspect that it is this feature of a constant magnitude of scalar gradient that allows stealth hair; thus, we look for an Einstein manifold, $R_{\mu \nu}=\Lambda g_{\mu \nu}$, with a scalar field satisfying $(\partial \phi)^{2}=X_{0}=$ const, under which assumptions the equations of motion become

$$
\begin{aligned}
& {\left[A_{3}\left(X_{0}\right)\left(\mathcal{E}_{3}-\Lambda X_{0}\right)-\left.2\left(K_{X}+4 \Lambda G_{X}\right)\right|_{X_{0}}\right] \phi_{\mu} \phi_{\nu}} \\
& \quad+\left.(K+2 \Lambda G)\right|_{X_{0}} g_{\mu \nu}=0
\end{aligned}
$$

for the metric and

$$
\begin{gathered}
A_{3}\left(X_{0}\right)\left(\mathcal{E}_{4}+2 R_{\mu \nu \rho \sigma} \phi^{\nu \sigma} \phi^{\mu} \phi^{\rho}-3 \Lambda X_{0} \square \phi\right) \\
-\left.2\left(K_{X}+4 \Lambda G_{X}\right)\right|_{X_{0}} \square \phi=0
\end{gathered}
$$

for the scalar, where for compactness we have defined

\footnotetext{
${ }^{1}$ These solutions were extended and generalized in different modified gravity theories with similar properties; see, for example, Refs. [11-15].
}

$$
\begin{aligned}
& \mathcal{E}_{3} \equiv(\square \phi)^{2}-\left(\phi_{\mu \nu}\right)^{2}, \\
& \mathcal{E}_{4} \equiv(\square \phi)^{3}-3 \square \phi\left(\phi_{\mu \nu}\right)^{2}+2\left(\phi_{\mu \nu}\right)^{3} .
\end{aligned}
$$

Although we are taking $\phi_{\mu}$ to have constant magnitude, $\phi_{\mu}$ is not itself necessarily constant; thus, $\mathcal{E}_{3,4}$ and $\square \phi$ can be spatially dependent, as, of course, is the Riemann tensor. Therefore, in order to satisfy Eq. (4), both the first and second terms must vanish. In other words, the magnitude of the gradient of $\phi, X_{0}$, must correspond to a zero of $A_{3}$, and the combination

$$
\left.\left(K_{X}+4 \Lambda G_{X}\right)\right|_{X_{0}}=0
$$

evaluated at $X_{0}$ must vanish. This is the required parametric constraint for the existence of our stealth hair. Equation (3) is then satisfied, provided we set $\Lambda=-K /\left.(2 G)\right|_{X_{0}}$; in other words, the cosmological constant appearing in the Einstein manifold is no longer the bare cosmological constant included in the constant part of the $K$ function but has been modified. This is the self-tuning property of these gravity theories. We emphasize that the above conditions need to be satisfied at the specific value of $X=X_{0}$, the magnitude of the constant gradient of $\phi$.

To sum up, given a general Lagrangian (1), we first look for zeros of $A_{3}$ that determine the value(s) of $X_{0}$, then ask that the derivatives of $G$ and $K$ are related at that specific value, $X_{0}$, according to (6). The effective cosmological constant is then fixed by the ratio of $K$ to $G$.

Having established the conditions under which Einsteinlike metrics could be solutions to modified gravity, we now make a key observation that allows us to explicitly construct a scalar field solution with the required property that its gradient has constant magnitude: given sufficient symmetry in a spacetime, the geodesic equation

$$
\frac{d^{2} x^{\mu}}{d \lambda^{2}}+\Gamma_{\rho \sigma}^{\mu} \frac{d x^{\rho}}{d \lambda} \frac{d x^{\sigma}}{d \lambda}=0
$$

can be solved using a Hamilton-Jacobi potential $S$, such that the gradient of the potential gives the tangent vector of the geodesic

$$
\frac{\partial S}{\partial x^{\mu}}=p_{\mu}=g_{\mu \nu} \frac{d x^{\nu}}{d \lambda} .
$$

Typically, this method is used to simplify the solution of a particular geodesic (such as the orbit of a planet); however, the form of the potential can be used over a wider range of coordinate values that in the case of a hypersurface orthogonal geodesic congruence becomes effectively the whole of the spacetime. Thus, given that $\phi_{\mu}$ has constant magnitude, as does the tangent vector of an affinely parametrized geodesic, it is natural to make the identification 


$$
\phi \leftrightarrow S
$$

the properties of the geodesic congruence then will ensure that $\phi$ has the requisite properties to be a stealth solution to the extended gravity equations of motion. Moreover, this provides a nice physical interpretation of the constants appearing in the solution.

Conversely, given that $(\partial \phi)^{2}$ is a constant, for convenience, rescale $\phi$ so that $X_{0}=1$, and then for clarity define a time coordinate $t \equiv \phi$ (if $\partial \phi$ is spacelike, then swap $t$ for $x$ in the following argument). The metric must therefore have the form

$$
d s^{2}=d t^{2}-\gamma_{i j}\left(d x^{i}-N_{i} d t\right)\left(d x^{j}-N_{j} d t\right) .
$$

Now, consider the "geodesic" equation for $\phi_{a}$ :

$$
\begin{aligned}
\phi^{a} \nabla_{a} \phi_{b}= & \phi^{a}\left[\partial_{a} \phi_{b}-\Gamma_{a b}^{c} \phi_{c}\right]=-\Gamma_{a b}^{t}\left(\delta_{t}^{a}+N^{j} \delta_{j}^{a}\right) \\
= & -\frac{1}{2}\left[-\left(N^{j} N_{j}\right)_{, b}+2 N^{j} N_{j, b}-N^{i} N^{j} \gamma_{i j, b}\right] \\
= & -\frac{1}{2}\left[-\left(N^{i} N^{j} \gamma_{i j}\right)_{, b}+2 N^{j}\left(\gamma_{i j} N^{i}\right)_{, b}\right. \\
& \left.-N^{i} N^{j} \gamma_{i j, b}\right]=0 .
\end{aligned}
$$

Thus, taking a constant gradient for $\phi$, and a $3+1$ coordinate system adapted to the scalar field, we can prove that $\partial \phi$ indeed satisfies the geodesic equation. Thus all stealth scalar solutions correspond to these geodesic congruences.

We will now illustrate this technique and find a rotating black hole with stealth hair. Consider the Kerr-(anti-)de Sitter [(A)dS] geometry [3]

$$
\begin{aligned}
d s^{2}= & -\frac{\Delta_{r}}{\Xi^{2} \rho^{2}}\left[d t-a \sin ^{2} \theta d \varphi\right]^{2}+\rho^{2}\left(\frac{d r^{2}}{\Delta_{r}}+\frac{d \theta^{2}}{\Delta_{\theta}}\right) \\
& +\frac{\Delta_{\theta} \sin ^{2} \theta}{\Xi^{2} \rho^{2}}\left[a d t-\left(r^{2}+a^{2}\right) d \varphi\right]^{2},
\end{aligned}
$$

where

$$
\begin{aligned}
& \Delta_{r}=\left(1-\frac{r^{2}}{\ell^{2}}\right)\left(r^{2}+a^{2}\right)-2 M r, \quad \Xi=1+\frac{a^{2}}{\ell^{2}}, \\
& \Delta_{\theta}=1+\frac{a^{2}}{\ell^{2}} \cos ^{2} \theta, \quad \rho^{2}=r^{2}+a^{2} \cos ^{2} \theta,
\end{aligned}
$$

$M$ is the black hole mass, $a$ is the angular momentum parameter, and $\ell=\sqrt{3 / \Lambda}$ is the de Sitter radius, related to the effective cosmological constant (for AdS, reverse the sign of $\ell^{2}$ ).

Applying the Hamilton-Jacobi technique, we note that the components of the metric (12) are independent of $t$ and $\varphi$; thus, $E=-p_{t}$ and $L_{z}=p_{\varphi}$ are two constants of the motion, identified with the energy and the azimuthal angular momentum, respectively. A third constant of motion is the magnitude of the tangent vector $g^{\mu \nu} p_{\mu} p_{\nu}=$ $X_{0}=-m^{2}$, associated with the rest mass of the test particle. ${ }^{2}$ Most importantly, however, a fourth constant was discovered by Carter [21] (here generalized to include $\Lambda$ ),

$$
\begin{aligned}
\mathcal{Q}= & \Delta_{\theta} p_{\theta}^{2}+m^{2} a^{2} \cos ^{2} \theta \\
& -\Xi^{2}\left[\left(a E-L_{z}\right)^{2}-\frac{\sin ^{2} \theta}{\Delta_{\theta}}\left(a E-\frac{L_{z}}{\sin ^{2} \theta}\right)^{2}\right],
\end{aligned}
$$

who demonstrated that the geodesic equation was separable. We can therefore write

$$
S=-E t+L_{z} \varphi+S_{r}(r)+S_{\theta}(\theta),
$$

where

$$
S_{r}= \pm \int \frac{\sqrt{R}}{\Delta_{r}} d r, \quad S_{\theta}= \pm \int \frac{\sqrt{\Theta}}{\Delta_{\theta}} d \theta
$$

with

$$
\begin{gathered}
R=\Xi^{2}\left[E\left(r^{2}+a^{2}\right)-a L_{z}\right]^{2} \\
-\Delta_{r}\left[\mathcal{Q}+\Xi^{2}\left(a E-L_{z}\right)^{2}+m^{2} r^{2}\right], \\
\Theta=-\Xi^{2} \sin ^{2} \theta\left(a E-\frac{L_{z}}{\sin ^{2} \theta}\right)^{2} \\
+\Delta_{\theta}\left[\mathcal{Q}+\Xi^{2}\left(a E-L_{z}\right)^{2}-m^{2} a^{2} \cos ^{2} \theta\right] .
\end{gathered}
$$

Now, let us look for explicit solutions for the scalar field $\phi=S$. This places further constraints on the potential, as we require $\phi_{\mu}$ to be regular throughout the spacetime. Checking regularity on the axes requires $\partial S / \partial \theta \rightarrow 0$ as $\theta \rightarrow 0, \pi$, i.e., $\Theta \propto \sin ^{2} \theta$. This in turn requires $L_{z}=0$ and $\mathcal{Q}+\Xi^{2} a^{2} E^{2}=m^{2} a^{2}$, and writing $\Xi E=\eta m$, we get

$$
\begin{aligned}
& \Theta=a^{2} m^{2} \sin ^{2} \theta\left(\Delta_{\theta}-\eta^{2}\right), \\
& R=m^{2}\left(r^{2}+a^{2}\right)\left(\eta^{2}\left(r^{2}+a^{2}\right)-\Delta_{r}\right) .
\end{aligned}
$$

This has now reduced the parameter space to an overall scaling, $m$, and a "relative energy" $\eta$, constrained to lie in $\eta \in\left[\eta_{c}, 1\right]$; the upper limit comes from $\Theta \geq 0$, and the lower limit comes from $R \geq 0$ in (19).

At first sight, it appears we have four distinct solutions coming from the choice of signs in (16); however, an interesting restriction occurs when $\eta=1$ or $\eta_{c}$. In this case, $\Theta$ (or $R$ ) vanishes for some value of $\theta$ (or $r$ ), and the branch choice changes. This is most easily seen for $\eta=1$; here, $\Theta_{1}=\frac{m^{2} a^{4}}{\ell^{2}} \sin ^{2} \theta \cos ^{2} \theta$, and the natural root is $\cos \theta$, which

\footnotetext{
${ }^{2}$ Note that for illustration we take timelike geodesics and, should a spacelike congruence be required, substitute $m^{2} \rightarrow-m^{2}$ in the derivation.
} 
changes sign across the hemisphere. The same phenomenon occurs for $R$, but this leads to an important consequence as we now discuss.

Inspection of (16) shows that $S_{r} \sim \pm m \eta r^{\star}$ near the event horizons, where $r^{\star}=\int d r\left(r^{2}+a^{2}\right) / \Delta_{r}$ is the tortoise coordinate; therefore, if we interpret $\sqrt{R}$ as being the positive root, our scalar field will be divergent at one or the other horizon (dependent on the branch choice). Note, however, that for $\eta_{c}, R$ has a quadratic zero at some $r_{0}$, $R \sim R^{\prime \prime}\left(r_{0}\right)\left(r-r_{0}\right)^{2} / 2$; thus, the true root, $\sqrt{R} \sim\left(r-r_{0}\right)$, changes sign at $r_{0}$. This means that for $\phi$ to be differentiable we must change the sign of $\sqrt{R}$ across $r_{0}$ and set

$$
S_{r}=\left(H\left[r-r_{0}\right]-H\left[r_{0}-r\right]\right) \int_{r_{0}}^{r} \frac{|\sqrt{R}| d r}{\Delta_{r}},
$$

where $H$ is the Heaviside step function. This now renders $\phi$ finite at both future event horizons and infinitely differentiable between the horizons as shown in Fig. 1.

It is worth emphasizing this last point: all black hole solutions in the literature for higher order scalar-tensor gravity are spherically symmetric and have scalar fields that diverge either on the black hole or cosmological event horizon. While this is not a physical problem when $\phi$ interacts with gravity only through its gradient, it is nonetheless a less appealing feature of these solutions. Here, we have constructed a rotating black hole with finite stealth scalar hair. This scalar will be manifestly continuous across each horizon and be straightforward to analyze in perturbation theory. Finally, the integral for the $\theta$-potential $S_{\theta}$ gives

$$
\begin{aligned}
\pm S_{\theta}= & \eta \log \left[\frac{\sqrt{1-\eta^{2}+\frac{a^{2}}{\ell^{2}} \cos ^{2} \theta}+\frac{a}{\ell} \cos \theta}{\sqrt{\left(1-\eta^{2}\right) \Delta_{\theta}}}\right] \\
& -\log \left[\frac{\sqrt{1-\eta^{2}+\frac{a^{2}}{\ell^{2}} \cos ^{2} \theta}+\frac{a}{\ell} \cos \theta}{\sqrt{1-\eta^{2}}}\right],
\end{aligned}
$$

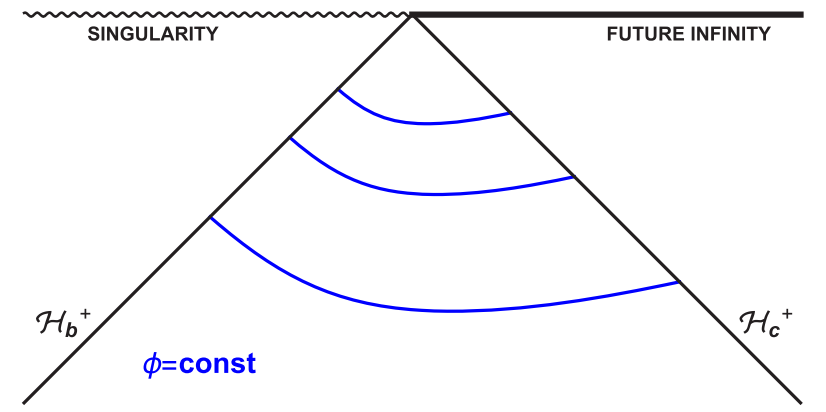

FIG. 1. Contours of constant $\phi$ for $a=G M=0.1 \ell, \eta_{c}=$ 0.612 in local Kruskal coordinates for the future event horizons, $\kappa_{b} U=-e^{-\frac{\kappa_{b}\left(t-r^{\star}\right)}{2}}, \kappa_{c} V=-e^{-\frac{\kappa_{c}\left(t+r^{*}\right)}{2}}\left(\kappa_{i}\right.$ being the absolute values of the surface gravities of each horizon).

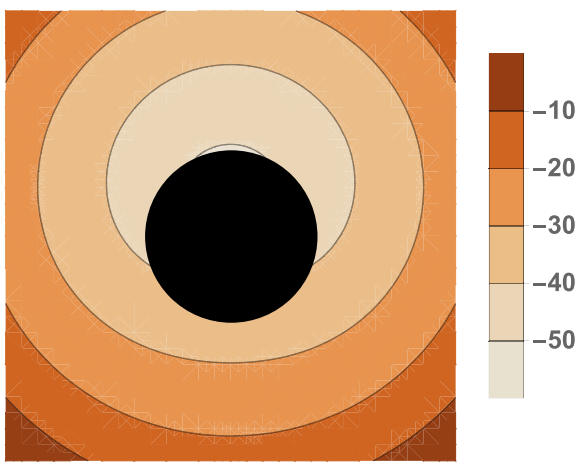

FIG. 2. Contours of $\phi$ at constant $v=t+r^{\star}$ in the $\{r, \theta\}$ plane near the black hole horizon with the same parameter values as in Fig. 1, taking $m=100$.

leading to an "off-center" behavior in the scalar as shown in Fig. 2.

For $\eta>\eta_{c}$, the radial function $R$ has no zero, and the scalar field diverges on one horizon, in common with the known solutions in the literature. The field also demonstrates a similar asymmetry in $\theta$, except for $\eta=1$, when

$$
S_{\theta}= \pm \frac{m \ell}{2} \log \Delta_{\theta}
$$

rendering the angular variation symmetric about the equator as shown in Fig. 3.

Our solution for $\phi$ shows a clear dependence on both $\theta$ and $r$ as well as the time dependence in common with known stealth solutions having spherical symmetry [10]. The key difference is that we can construct a scalar that is finite on both the black hole and cosmological horizon. To compare to solutions in the literature, we take spherical symmetry $(a \rightarrow 0)$ and find general solutions of the form $\phi=-m \eta t \pm S_{r}$. Once again, we have a finite- $\phi$ solution for $\eta_{c}^{2}=1-3(M / \ell)^{2 / 3}$,

$$
\phi=-m\left[\eta_{c} t+\int \frac{r\left(r-r_{0}\right) \sqrt{r\left(r+2 r_{0}\right)}}{\ell \Delta_{r}} d r\right]
$$

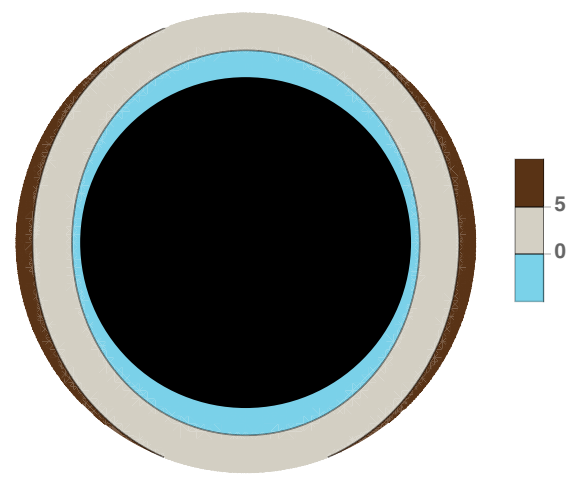

FIG. 3. Contours of $\phi$ for $\eta=1$ at constant $v=t+r^{\star}$ in the $\{r, \theta\}$ plane $(a=G M=0.22 \ell, m=100)$. 
where $r_{0}=M^{1 / 3} \ell^{2 / 3}$. It is interesting to compare this to the time-dependent solution of a black hole in slow-roll inflation [22,23]. There, the scalar profile $\phi_{S R} \propto T$ (for a suitable time coordinate) is also finite at both horizons, but (23) has constant gradient, whereas $\phi_{S R}$ solves a wave equation, resulting in a slightly different radial profile between the horizons.

Also, note that in the case of spherical symmetry we can relax our constraint $A_{3}\left(X_{0}\right)=0$. In this case, $\mathcal{E}_{3}, \mathcal{E}_{4}$, and the Riemann tensor term appearing in $(3,4)$ have a simple form and combine to require

$$
\left.\left(K_{X}+4 \Lambda G_{X}+\frac{3}{2} \Lambda X A_{3}\right)\right|_{X_{0}}=0
$$

with the same self-tuning condition for the cosmological constant $^{3}$; however, note that $A_{3}\left(X_{0}\right) \neq 0$ requires $\eta \equiv 1$, and thus we no longer have the $\eta$ degree of freedom. What now happens is that spacetime becomes foliated by surfaces of constant $\phi$ that are flat (for $\Lambda=0$ ).

Another interesting possibility for spherical symmetry is a static solution, found by setting $E=0$; hence, $X_{0}>0$, and $\phi$ corresponds to a congruence of spacelike geodesics,

$$
\phi_{s}=S_{r}(r)=\sqrt{X_{0}} \int \frac{r}{\sqrt{\Delta_{r}}} d r
$$

agreeing with a solution reported in Ref. [20].

It is also worth noting a side result of our analysis: a search for solutions with $X=X_{0}$ and spherical symmetry in $c_{T}=1$ theories (1) allows only for Einstein geometries. This is unlike Horndeski theories (with $c_{T} \neq 1$ ) where solutions of black holes and solitons that have $X=X_{0}$ but are not $t$ Einstein spaces were found (for a concise up-to-date review, see Ref. [25]). This is an interesting feature, hinting that solutions of $c_{T}=1$ theories, where $X$ is not constant, belong to branches that eventually flow toward $X$ constant solutions with an Einstein space metric.

\footnotetext{
${ }^{3}$ See Ref. [24] for a precise analysis of self-tuning conditions in spherical symmetry.
}

To sum up, we have presented the first exact solutions for a rotating black hole with scalar hair in shift-symmetric scalar-tensor theories with unitary speed for gravitational waves. Our method was based on an interesting correspondence between families of black hole geodesics and stealth solutions in modified gravity. The geodesic correspondence can be used to find other stealth solutions, for instance, for more general type D spacetimes, or even nonstealth solutions that do not exist in GR-one only needs to compute the Hamilton-Jacobi potential for a geodesic in the relevant spacetime. It is also plausible that this technique can be extended to other modifications of gravity, such as vector-tensor theories where we know that certain stealth solutions exist. Although we did not consider charged black holes, clearly one can also use this method to find stealth Kerr-Newman solutions. Perhaps most importantly, we have presented a scalar solution that is finite at both the cosmological and event horizons, thus manifestly extendible beyond the cosmological horizon. The angular asymmetry of this solution could provide a distinctive signature of this hair, although this would require a full perturbation analysis beyond the scope of this investigation.

\section{ACKNOWLEDGMENTS}

It is a pleasure to thank Eugeny Babichev, Gilles EspositoFarèse, Antoine Lehébel, Karim Noui, Eric Gourgoulhon, and Karim Van Aelst for many interesting discussions. C. C. and N. S. would like to acknowledge networking support by the GWverse COST Action CA16104, Black holes, gravitational waves and fundamental physics. C. C. acknowledges support from the CNRS Grant No. 80PRIME and thanks the Laboratory of Astronomy of AUTh in Thessaloniki for hospitality during the course of this work. M. C. is supported by the Labex P2IO and the Enhanced Eurotalents Fellowship. R. G. is supported in part by the STFC (consolidated Grant No. ST/P000371/1) and in part by the Perimeter Institute. Research at Perimeter Institute is supported by the Government of Canada through the Department of Innovation, Science and Economic Development and by the Province of Ontario through the Ministry of Research and Innovation.
[1] B. P. Abbott et al. (LIGO Scientific and Virgo Collaborations), Observation of Gravitational Waves from a Binary Black Hole Merger, Phys. Rev. Lett. 116, 061102 (2016).

[2] B. Carter, Hamilton-Jacobi and Schrodinger separable solutions of Einstein's equations, Commun. Math. Phys. 10, 280 (1968).

[3] B. Carter, A new family of einstein spaces, Phys. Lett. 26A, 399 (1968).
[4] S. Doeleman et al., Imaging an Event Horizon: SubmmVLBI of a Super Massive Black Hole, in Astro1010: The Astronomy and Astrophysics Decadal Survey, 68, arXiv: 0906.3899.

[5] T. Johannsen, C. Wang, A. E. Broderick, S. S. Doeleman, V.L. Fish, A. Loeb, and D. Psaltis, Testing General Relativity with Accretion-Flow Imaging of Sgr A*, Phys. Rev. Lett. 117, 091101 (2016). 
[6] D. Langlois and K. Noui, Degenerate higher derivative theories beyond Horndeski: Evading the Ostrogradski instability, J. Cosmol. Astropart. Phys. 02 (2016) 034.

[7] M. Crisostomi, K. Koyama, and G. Tasinato, Extended scalar-tensor theories of gravity, J. Cosmol. Astropart. Phys. 04 (2016) 044.

[8] J. Ben Achour, M. Crisostomi, K. Koyama, D. Langlois, K. Noui, and G. Tasinato, Degenerate higher order scalartensor theories beyond Horndeski up to cubic order, J. High Energy Phys. 12 (2016) 100.

[9] B. P. Abbott et al., Multi-messenger observations of a binary neutron star merger, Astrophys. J. 848, L12 (2017).

[10] E. Babichev and C. Charmousis, Dressing a black hole with a time-dependent Galileon, J. High Energy Phys. 08 (2014) 106.

[11] M. Rinaldi, Black holes with non-minimal derivative coupling, Phys. Rev. D 86, 084048 (2012).

[12] T. Kobayashi and N. Tanahashi, Exact black hole solutions in shift symmetric scalar-tensor theories, Prog. Theor. Exp. Phys. 2014, 73E02 (2014).

[13] E. Babichev, C. Charmousis, and A. Lehébel, Asymptotically flat black holes in Horndeski theory and beyond, J. Cosmol. Astropart. Phys. 04 (2017) 027.

[14] J. Chagoya, G. Niz, and G. Tasinato, Black holes and Abelian symmetry breaking, Classical Quantum Gravity 33, 175007 (2016).

[15] E. Babichev, C. Charmousis, and M. Hassaine, Black holes and solitons in an extended Proca theory, J. High Energy Phys. 05 (2017) 114.
[16] E. Babichev, C. Charmousis, G. Esposito-Farèse, and A. Lehébel, Stability of Black Holes and the Speed of Gravitational Waves within Self-Tuning Cosmological Models, Phys. Rev. Lett. 120, 241101 (2018).

[17] E. Babichev, C. Charmousis, G. Esposito-Farèse, and A. Lehébel, Hamiltonian unboundedness vs stability with an application to Horndeski theory, Phys. Rev. D 98, 104050 (2018).

[18] J. Chagoya and G. Tasinato, Compact objects in scalartensor theories after GW170817, J. Cosmol. Astropart. Phys. 08 (2018) 006.

[19] J. Ben Achour and H. Liu, Hairy Schwarzschild-(A)dS black hole solutions in DHOST theories beyond shift symmetry, Phys. Rev. D 99, 064042 (2019).

[20] H. Motohashi and M. Minamitsuji, Exact black hole solutions in shift-symmetric quadratic DHOST theories, Phys. Rev. D 99, 064040 (2019).

[21] B. Carter, Global structure of the Kerr family of gravitational fields, Phys. Rev. 174, 1559 (1968).

[22] S. Chadburn and R. Gregory, Time dependent black holes and scalar hair, Classical Quantum Gravity 31, 195006 (2014).

[23] R. Gregory, D. Kastor, and J. Traschen, Evolving black holes in inflation, Classical Quantum Gravity 35, 155008 (2018).

[24] E. Babichev and G. Esposito-Farèse, Cosmological selftuning and local solutions in generalized Horndeski theories, Phys. Rev. D 95, 024020 (2017).

[25] A. Lehébel, Compact astrophysical objects in modified gravity, arXiv:1810.04434. 